\title{
A Simplified Predictive Control of Constrained Markov Jump System with Mixed Uncertainties
}

\author{
Yanyan Yin, ${ }^{1}$ Yanqing Liu, ${ }^{1}$ and Hamid R. Karimi ${ }^{2}$ \\ ${ }^{1}$ Key Laboratory of Advanced Process Control for Light Industry (Ministry of Education), Institute of Automation, \\ Jiangnan University, Wuxi 214122, China \\ ${ }^{2}$ Department of Engineering, Faculty of Engineering and Science, University of Agder, 4898 Grimstad, Norway
}

Correspondence should be addressed to Yanyan Yin; yanyan0510@gmail.com

Received 17 November 2013; Revised 27 January 2014; Accepted 14 February 2014; Published 26 March 2014

Academic Editor: Shuping He

Copyright (C) 2014 Yanyan Yin et al. This is an open access article distributed under the Creative Commons Attribution License, which permits unrestricted use, distribution, and reproduction in any medium, provided the original work is properly cited.

\begin{abstract}
A simplified model predictive control algorithm is designed for discrete-time Markov jump systems with mixed uncertainties. The mixed uncertainties include model polytope uncertainty and partly unknown transition probability. The simplified algorithm involves finite steps. Firstly, in the previous steps, a simplified mode-dependent predictive controller is presented to drive the state to the neighbor area around the origin. Then the trajectory of states is driven as expected to the origin by the final-step modeindependent predictive controller. The computational burden is dramatically cut down and thus it costs less time but has the acceptable dynamic performance. Furthermore, the polyhedron invariant set is utilized to enlarge the initial feasible area. The numerical example is provided to illustrate the efficiency of the developed results.
\end{abstract}

\section{Introduction}

Hybrid systems are a class of dynamical systems denoted by an interaction between the continuous and discrete dynamics. In control community, the researchers tend to view hybrid systems as continuous state and discrete switching which focuses on the continuous state of dynamic system. Switched systems are a natural result from this point of view. Since switching systems can be applied to model the systems involving abrupt sudden changes which are widely found in the systems of economics and communications as well as manufacturing, more attention has been paid to them (see robust stabilization [1], finite-time analysis [2] and asynchronous switching [3]). When the system model is linear and the switching is driven by Markov process, it leads to Markov jump linear system (MJS). Specifically, MJS presents a stochastic Markov chain to describe the random changes of system parameters or structures, where the dynamic of MJS is switching among the models governed by a finite Markov chain. Due to this superiority, MJS has been widely investigated during the last twenty years. Attractive pioneer works have been obtained (see controller design [4], 2D MJS control [5], peak-to-peak filtering [6], and finite-time control $[7,8])$. However, the cases of completely known transition probability (TP) considered in [4-8] are not always achievable since the TP is not easy to be fully accessible (see the delay or packet loss in networked control systems [9]). Thus it is necessary to investigate the partly unknown case $[10-12]$.

On the other hand, the systems in practice are usually subject to input/output constraints. Thus, model predictive control (MPC) is then introduced to solve the problem of MJS with constraints since MPC can explicitly solve the constraints in control action. Successful MPC application in discrete-time MJS can be obtained in [13, 14]. Normally, MPC is reformulated as online quadratic program and results have been reported (see stability $[15,16]$ and enlarged terminal sets [17]). It should be noted that the online computation in the literature [15-17] leads to heavy computational burden. Thus, the researchers attempted to try a new alternative method to solve the problem. For this reason, explicit MPC [18] is presented. However, when the size of system increases, the time of searching explicit MPC law will also increase sharply.

Based on the above analysis, a simplified MPC design framework is introduced to reduce the burden of online 
computation for the constrained MJS with mixed uncertainties. The basic idea is that $(N-1)$ steps of modedependent MPC are designed to steer the state to a final neighbour area which includes the origin. Then the final step of robust mode-independent MPC is devised to force the state towards the origin regardless of model uncertainty and transition probability uncertainty. This simplified MPC dramatically reduces the burden of computation with minor performance loss, which implies good balance between the calculation time and dynamical performance. Furthermore, the polyhedron invariant set is applied to further enlarge the initial feasible area.

The construction of the paper is as follows. Section 2 gives the basic dynamical of the system. Section 3 gives the finitestep simplified MPC algorithm and it is formulated as LMIs. Section 4 presents a numerical example to show the efficiency of the results. Section 5 concludes the paper.

Notations. The notations are as follows: $R^{n}$ denotes a $n$ dimensional Euclidean space, $A^{T}$ stands for the transpose of a matrix, $E\{\cdot\}$ denotes the expectation of the stochastic process or vector, a positive-definite matrix is described as $P>0$, $I$ means the unit matrix with appropriate dimension, and $*$ means the symmetric term in a symmetric matrix.

\section{Problem Statement and Preliminaries}

The constrained discrete-time MJSs with mixed uncertainties are considered in this paper:

$$
\begin{gathered}
x_{k+1}=A\left(r_{k}\right) x_{k}+B\left(r_{k}\right) u_{k}, \\
y_{k}=C\left(r_{k}\right) x_{k},
\end{gathered}
$$

where $x_{k} \in R^{n_{x}}, u_{k} \in R^{n_{u}}, y_{k} \in R^{n_{y}}$, respectively, denote the state vector, the input vector, and the controlled output vector. The discrete-time Markov stochastic process $\left\{r_{k}, k \geq 0\right\}$ takes values in a finite set $\Gamma$, where $\Gamma$ contains $\sigma$ modes of system (1), $\Gamma=\{1,2,3, \ldots, \sigma\}$, and $r_{0}$ represents the initial mode. The uncertain system model $A\left(r_{k}\right)$ and $B\left(r_{k}\right)$ belong to the model sets

$$
\begin{gathered}
\Omega\left(r_{k}\right)=\left\{\left[A\left(r_{k}\right), B\left(r_{k}\right)\right], A\left(r_{k}\right)=\sum_{l=1}^{L} \alpha_{\iota} A_{\iota}\left(r_{k}\right),\right. \\
\left.B\left(r_{k}\right)=\sum_{l=1}^{L} \alpha_{\iota} B_{l}\left(r_{k}\right), \sum_{l=1}^{L} \alpha_{\iota}=1\right\} .
\end{gathered}
$$

Inputs and outputs constraints are subject to

$$
\begin{aligned}
& -u_{\lim } \leq u_{k} \leq u_{\lim }, \\
& -y_{\lim } \leq y_{k} \leq y_{\lim } .
\end{aligned}
$$

The transition probability (TP) matrix is denoted by $\Pi(k)=$ $\left\{\pi_{i j}(k)\right\}, i, j \in \Gamma$, where $\pi_{i j}(k)=P\left(r_{k+1}=j \mid r_{k}=i\right)$ is the transition probability from mode $i$ at time $k$ to mode $j$ at time $k+1$. The elements in TP matrix satisfy $\pi_{i j}(k) \geq 0$ and $\sum_{j=1}^{\sigma} \pi_{i j}(k)=1$ :

$$
\pi=\left[\begin{array}{cccc}
\pi_{11} & \pi_{12} & \ldots & \pi_{1 \sigma} \\
\pi_{21} & \pi_{22} & \ldots & \pi_{2 \sigma} \\
\vdots & \vdots & \ddots & \vdots \\
\pi_{\sigma 1} & \pi_{\sigma 2} & \ldots & \pi_{\sigma \sigma}
\end{array}\right]
$$

The uncertain transition probability (TP) implies that some elements in $\pi$ are unknown; a four-mode transition probability (TP) matrix $\pi$ may be

$$
\pi=\left[\begin{array}{cccc}
? & \pi_{12} & ? & ? \\
\pi_{21} & \pi_{22} & ? & ? \\
\pi_{31} & ? & ? & ? \\
? & ? & \pi_{43} & ?
\end{array}\right]
$$

where "?" represents the inaccessible element in TP matrix. For convenience, we denote $\pi=\pi_{r_{k}}^{k}+\pi_{r_{k}}^{u k}$, for all mode $r_{k} \in \Gamma$ at sampling time $k$, if $\pi_{r_{k}}^{k} \neq 0$, and redescribe it as $\pi_{r_{k}}^{k}=\left(\kappa_{r_{k}}^{1}, \ldots, \kappa_{r_{k}}^{\tau}\right)$, for all $1 \leq l \leq \tau$, where $\kappa_{r_{k}}^{l}$ represents the $l$ th exact element in the $i$ th row of $\pi, \Pi_{r_{k}}^{k}=\sum_{j \in \pi_{r_{k}}^{k}} \pi_{r_{k} r_{k+1}}$.

Some preliminaries are introduced before proceeding.

Definition 1 (see [6]). For any initial mode $r_{0}$ and state $x_{0}$, discrete-time MJS (1) is said to be stochastically stable if

$$
\lim _{k \rightarrow \infty} E\left\{x_{k}^{T} x_{k} \mid x_{0}, r_{0}\right\} \rightarrow 0
$$

Definition 2. For MJS (1), an ellipsoid set $\Theta=\left\{x \in R^{n_{x}} \mid\right.$ $\left.x_{k}^{T} P_{k}\left(r_{k}\right) x_{k} \leq \gamma_{k}\right\}$ associated with the state is said to be asymptotically mode-dependent stable, if the following holds, whenever $x_{k_{0}} \in \Theta$, then $x_{k} \in \Theta$ for $k \geq k_{0}$ and $x_{k} \rightarrow 0$ when $k \rightarrow \infty$.

Next, we first derive the online optimal MPC algorithm for system (1). The aim is to minimize the function cost related to worst-case performance and then in Section 4 the corresponding simplified MPC algorithm will be derived. Finally the polyhedron invariant set is applied to further improve the initial feasible district.

\section{Simplified MPC Design}

\subsection{Online Optimal MPC}

Theorem 3. Consider MJS (1) with model uncertainties (2) and partly unknown TP matrix (6), at sampling time $k$, if there exist a set of matrices $F_{k}\left(r_{k}\right)$, such that the following holds:

$$
\min _{F_{k}\left(r_{k}\right)} \max _{A_{\iota}\left(r_{k}\right), B_{t}\left(r_{k}\right), \pi_{r_{k} r_{k+1}}, r_{k}, r_{k+1} \in \Gamma} J_{\infty}(k)
$$


s.t.

$$
\begin{gathered}
-u_{\lim } \leq u_{k} \leq u_{\lim }, \\
-y_{\lim } \leq y_{k} \leq y_{\lim }, \\
E\left\{V\left(x_{k+1}, r_{k+1} \mid x_{0}, r_{0}\right)\right\}-E\left\{V\left(x_{k}, r_{k} \mid x_{0}, r_{0}\right)\right\} \\
\leq-E\left\{x_{k}^{T} Q\left(r_{k}\right) x_{k}+u_{k}^{T} R\left(r_{k}\right) u_{k} \mid x_{0}, r_{0}\right\} .
\end{gathered}
$$

Then, it decides an upper bound on $J_{\infty}(k)$, where $u_{k}=$ $F_{k}\left(r_{k}\right) x_{k}, J_{\infty}(k)=E\left\{\sum_{k=0}^{\infty}\left(x_{k}^{T} Q\left(r_{k}\right) x_{k}+u_{k}^{T} R\left(r_{k}\right) u_{k}\right) \mid x_{0}, r_{0}\right\}$, $\mathrm{Q}\left(r_{k}\right), R\left(r_{k}\right)$ are positive definite weighting matrices.

Proof. It is assumed that at the sampling time $k$, a statefeedback law $u(k+i \mid k)=F_{k}\left(r_{k}\right) x(k+i \mid k)$, is applied to minimize the worst cost function of $J_{k}$; it is easy to show that $V\left(x_{k}, r_{k} \mid x_{0}, r_{0}\right)$ is an upper bound on $J_{\infty}(k)$. Let $V\left(x_{k}\right)=$ $x_{k}^{T} P_{k}\left(r_{k}\right) x_{k}, P_{k}\left(r_{k}\right)>0$, be a quadratic Lyapunov function. For any $\left[A_{t}\left(r_{k}\right), B_{t}\left(r_{k}\right) \in \Omega\left(r_{k}\right)\right]$, the following constraint holds

$$
\begin{gathered}
E\left\{V\left(x_{k+1}, r_{k+1} \mid x_{0}, r_{0}\right)\right\}-E\left\{V\left(x_{k}, r_{k} \mid x_{0}, r_{0}\right)\right\} \\
\leq-E\left\{x_{k}^{T} Q\left(r_{k}\right) x_{k}+u_{k}^{T} R\left(r_{k}\right) u_{k} \mid x_{0}, r_{0}\right\} .
\end{gathered}
$$

Summing (12) from $i=0$ to $\infty$ on both sides and using the fact $x_{k \rightarrow \infty}=0$ or $V\left(x_{k \rightarrow \infty}\right)=0$, we obtain

$$
J_{\infty}(k) \leq V\left(x_{k}, r_{k} \mid x_{0}, r_{0}\right)=x_{k}^{T} P_{k}\left(r_{k}\right) x_{k}
$$

which implies that $V\left(x_{k}, r_{k} \mid x_{0}, r_{0}\right)$ is an upper bound on $J_{\infty}(k)$.

Theorem 4. Consider MJS (1) with polytope model uncertainties (2) and partly unknown TP matrix (4), if there exist a set of positive definite matrices $X_{k}\left(r_{k}\right), Y_{k}\left(r_{k}\right)$, such that the following optimization problem (12) has an optimal solution:

$$
\min _{F_{k}\left(r_{k}\right)} \max _{A_{\iota}\left(r_{k}\right), B_{t}\left(r_{k}\right), \pi_{r_{k} r_{k+1}}, r_{k}, r_{k+1} \in \Gamma} \gamma_{k}
$$

s.t.

$$
\begin{aligned}
& {\left[\begin{array}{cc}
1 & * \\
x_{k} & X_{k}\left(r_{k}\right)
\end{array}\right] \geq 0, \quad \forall r_{k} \in \Gamma, r_{k+1} \in \pi_{r_{k+1}}^{u k},} \\
& {\left[\begin{array}{cc}
Z & Y_{k}\left(r_{k}\right) \\
* & X_{k}\left(r_{k}\right)
\end{array}\right] \geq 0, \quad Z_{t t} \leq\left(u_{\lim }^{t}\right)^{2},} \\
& {\left[\begin{array}{cc}
X_{k}\left(r_{k}\right) & * \\
C\left(r_{k}\right) \theta_{l}\left(r_{k}\right) & M
\end{array}\right] \geq 0, \quad M_{h h} \leq\left(y_{\text {lim }}^{h}\right)^{2},} \\
& {\left[\begin{array}{cc}
X_{k}\left(r_{k}\right) & * \\
\theta_{l}\left(r_{k}\right) & X_{k}\left(r_{k+1}\right)
\end{array}\right] \geq 0, \quad \forall r_{k+1} \in \pi_{r_{k}}^{u k},} \\
& {\left[\begin{array}{ccccc}
\Pi_{r_{k}}^{k} X_{k}\left(r_{k}\right) & U^{T}\left(r_{k}\right) & X_{k}\left(r_{k}\right) Q^{1 / 2}\left(r_{k}\right) & Y_{k}^{T}\left(r_{k}\right) Q^{1 / 2}\left(r_{k}\right) \\
* & W\left(r_{k+1}\right) & 0 & 0 & \\
* & * & \gamma_{k} I & 0 & \\
* & * & * & \gamma_{k} I &
\end{array}\right] \geq 0,}
\end{aligned}
$$

then, the mode-dependent state-feedback which minimizes the upper bound $\gamma_{k}$ on $J_{\infty}(k)$ and simultaneously stabilizes the closed-loop system within an ellipsoid $\varepsilon=\left\{x_{k}^{T} X_{k}^{-1}\left(r_{k}\right) x_{k} \leq 1\right\}$ is calculated by $u(k+i \mid k)=F_{k}\left(r_{k+i}\right) x_{k+i \mid k}, F_{k}\left(r_{k+i}\right)=$ $Y_{k}\left(r_{k+i}\right) X_{k}^{-1}\left(r_{k+i}\right)$, where $X_{k}\left(r_{k}\right)=\gamma_{k} P_{k}^{-1}\left(r_{k}\right), \theta_{l}\left(r_{k}\right)=$ $A_{l}\left(r_{k}\right) X_{k}\left(r_{k}\right)+B_{l}\left(r_{k}\right) Y_{k}\left(r_{k}\right), U^{T}\left(r_{k}\right)=\left[\sqrt{\kappa_{r_{k}}^{1}} \theta_{l}^{T}\left(r_{k}\right), \ldots\right.$, $\left.\sqrt{\kappa_{r_{k}}^{\tau}} \theta_{l}^{T}\left(r_{k}\right)\right], W\left(r_{k+1}\right)=\operatorname{diag}\left\{X_{k}\left(\kappa_{r_{k}}^{1}\right), X_{k}\left(\kappa_{r_{k}}^{2}\right), \ldots, X_{k}\left(\kappa_{r_{k}}^{\tau}\right)\right\}$, $Z_{t t}, M_{h h}$, respectively, describe the $t$ th, hth diagonal element of $Z, M, u_{\mathrm{lim}}^{t}$ and $y_{\mathrm{lim}}^{h}$, respectively, describe the th and $h$ th element of input and output constraints, $t=1,2, \ldots, n_{u}, h=$ $1,2, \ldots, n_{y}$.

Proof. Let $X_{k}\left(r_{k}\right)=\gamma_{k} P_{k}^{-1}\left(r_{k}\right) ; J_{\infty}(k) \leq \gamma_{k}$ in (13) can be solved by the following LMIs:

$$
\begin{array}{r}
{\left[\begin{array}{cc}
1 & * \\
x_{k} & X_{k}\left(r_{k}\right)
\end{array}\right] \geq 0,} \\
\forall r_{k} \in \Gamma, \quad r_{k+1} \in \pi_{r_{k+1}^{u k}} .
\end{array}
$$

The input/output constraints are guaranteed by (16) and (17); the proof is similar to [19]; here we omit the proof. Equation (11) is equivalent to

$$
\begin{aligned}
\Xi\left(r_{k}\right)= & P_{k}\left(r_{k}\right)-\theta_{l}^{T}\left(r_{k}\right)\left(\sum_{r_{k+1} \in \pi} \pi_{r_{k} r_{k+1}} P_{k}\left(r_{k+1}\right)\right) \\
& \times \theta_{l}\left(r_{k}\right)-Q\left(r_{k}\right)-F_{k}^{T}\left(r_{k}\right) R\left(r_{k}\right) F_{k}\left(r_{k}\right) \geq 0 .
\end{aligned}
$$

Since $\sum_{r_{k+1} \in \pi} \pi_{r_{k} r_{k+1}}=1, \pi_{r_{k} r_{k+1}} \geq 0, \Pi_{k}^{r_{k}}=\sum_{r_{k+1} \in \pi_{r_{k}}^{k}} \pi_{r_{k} r_{k+1}}$, it leads to

$$
\begin{aligned}
\Xi\left(r_{k}\right)= & \left(\sum_{r_{k+1} \in \pi} \pi_{r_{k} r_{k+1}}\right) P_{k}\left(r_{k}\right)-\theta_{l}^{T}\left(r_{k}\right) \\
& \times\left(\sum_{r_{k+1} \in \pi} \pi_{r_{k} r_{k+1}} P_{k}\left(r_{k+1}\right)\right) \theta_{l}\left(r_{k}\right)-Q\left(r_{k}\right) \\
& -F_{k}^{T}\left(r_{k}\right) R\left(r_{k}\right) F_{k}\left(r_{k}\right) \\
= & \Pi_{r_{k}}^{k} P\left(r_{k}\right)-\theta_{l}^{T}\left(r_{k}\right) \\
& \times\left(\sum_{r_{k+1} \in \pi_{r_{k}}^{k}} \pi_{r_{k} r_{k+1}} P_{k}\left(r_{k+1}\right)\right) \theta_{l}\left(r_{k}\right) \\
& +\left(\sum_{r_{k+1} \in \pi_{r_{k}}^{u k}} \pi_{r_{k} r_{k+1}}\right) \\
& \times\left(P_{k}\left(r_{k}\right)-\theta_{l}^{T}\left(r_{k}\right) P_{k}\left(r_{k+1}\right) \theta_{l}\left(r_{k}\right)\right) \\
& -Q\left(r_{k}\right)-F_{k}^{T}\left(r_{k}\right) R\left(r_{k}\right) F_{k}\left(r_{k}\right) \geq 0 .
\end{aligned}
$$


One sufficient condition to ensure (22) is

$$
\begin{gathered}
\Pi_{r_{k}}^{k} P_{k}\left(r_{k}\right)-\theta_{l}^{T}\left(r_{k}\right)\left(\sum_{r_{k+1} \in \pi_{r_{k}}^{k}} \pi_{r_{k} r_{k+1}} P_{k}\left(r_{k+1}\right)\right) \\
\times \theta_{l}\left(r_{k}\right)-Q\left(r_{k}\right)-F_{k}^{T}\left(r_{k}\right) R\left(r_{k}\right) F_{k}\left(r_{k}\right) \geq 0, \\
P_{k}\left(r_{k}\right)-\theta_{l}^{T}\left(r_{k}\right) P_{k}\left(r_{k+1}\right) \theta_{l}\left(r_{k}\right) \geq 0 .
\end{gathered}
$$

Considering the Schur theory complement lemma, (16) and (17) can be derived.

Actually the feedback controller can make the closedloop system stable in the ellipsoid $\varepsilon=\left\{x_{k}^{T} X_{k}^{-1}\left(r_{k}\right) x_{k} \leq 1\right\}$. Assume that the optimal $P_{k}^{*}\left(r_{k}\right), F_{k}^{*}\left(r_{k}\right)$ at the moment $k$ are

$$
\begin{gathered}
P_{k}^{*}\left(r_{k}\right)=\gamma_{k}^{*}\left(X_{k}^{*}\left(r_{k}\right)\right)^{-1}, \\
F_{k}^{*}\left(r_{k}\right)=Y_{k}^{*}\left(X_{k}^{*}\left(r_{k}\right)\right)^{-1}, \\
\theta_{l k}^{*}\left(r_{k}\right)=A_{l}\left(r_{k}\right)+B_{l}\left(r_{k}\right) F_{k}^{*}\left(r_{k}\right), \\
\vartheta_{l k}^{*}\left(r_{k}\right)=A_{l}\left(r_{k}\right) X_{k}^{*}\left(r_{k}\right)+B_{l}\left(r_{k}\right) Y_{k}^{*}\left(r_{k}\right) .
\end{gathered}
$$

Equations (18) and (19) lead to

$$
\begin{aligned}
x_{k}^{T} P_{k}^{*}\left(r_{k}\right) x_{k} \geq & x_{k}^{T}\left(\theta_{l k}^{*}\left(r_{k}\right)\right)^{T} \\
& \times \sum_{r_{k+1} \in \pi} \pi_{r_{k} r_{k+1}} P\left(r_{k+1}\right) \theta_{l k}^{*}\left(r_{k}\right) x_{k} \\
& +x_{k}^{T} Q\left(r_{k}\right) x_{k}+x_{k}^{T} F_{k}^{T}\left(r_{k}\right) R\left(r_{k}\right) F_{k}\left(r_{k}\right) x_{k}, \\
E\left\{x_{k}^{T} P_{k}^{*}\left(r_{k}\right) x_{k}\right\} & \\
\geq E\left\{x_{k+1}^{T} P_{k}^{*}\left(r_{k+1}\right) x_{k+1}\right\} & \\
& +x_{k}^{T} Q\left(r_{k}\right) x_{k}+x_{k}^{T} F_{k}^{T}\left(r_{k}\right) R\left(r_{k}\right) F_{k}\left(r_{k}\right) x_{k} .
\end{aligned}
$$

$P_{k+1}^{*}\left(r_{k+1}\right)$ is the optimal value at moment $k+1 ; P_{k}^{*}\left(r_{k+1}\right)$ is a feasible one at moment $k+1$. By the optimum definition,

$$
x_{k+1}^{T} P_{k}^{*}\left(r_{k+1}\right) x_{k+1} \geq x_{k+1}^{T} P_{k+1}^{*}\left(r_{k+1}\right) x_{k+1} ;
$$

then,

$$
\begin{aligned}
E\left\{x_{k}^{T} P_{k}^{*}\left(r_{k}\right) x_{k}\right\} & \\
\geq & E\left\{x_{k+1}^{T} P_{k+1}^{*}\left(r_{k+1}\right) x_{k+1}\right\} \\
& +x_{k}^{T} Q\left(r_{k}\right) x_{k}+x_{k}^{T} F_{k}^{T}\left(r_{k}\right) R\left(r_{k}\right) F_{k}\left(r_{k}\right) x_{k} .
\end{aligned}
$$

It is shown that $E\left\{x_{k}^{T} P_{k}^{*}\left(r_{k}\right) x_{k}\right\}$ decrease strictly as $E\left\{x_{k}^{T} \Phi_{k}^{*}\left(r_{k}\right) x_{k}\right\} \rightarrow 0, k \rightarrow \infty$

From Definition 1, the system is stochastically stable. From (27), then

$$
E\left\{x_{k}^{T} P_{k}^{*}\left(r_{k}\right) x_{k}\right\} \geq E\left\{x_{k+1}^{T} P_{k+1}^{*}\left(r_{k+1}\right) x_{k+1}\right\} .
$$

This implies that the ellipsoid is an asymptotically stable invariant one, which completes the proof.
Corollary 5. Consider MJS (1) with model uncertainties (2) and TP matrix (4) at current moment $k$; supposing that there exists a set of positive definite matrices $X, Y$, such that the following optimization problem has an optimal solution:

$$
\min _{\gamma_{k}, X, Y} \gamma_{k}
$$

s.t.

$$
\begin{aligned}
& {\left[\begin{array}{cc}
1 & * \\
x_{k} & X
\end{array}\right] \geq 0} \\
& \forall r_{k} \in \Gamma, \quad r_{k+1} \in \pi_{r_{k+1}}^{u k}, \\
& {\left[\begin{array}{ll}
Z & Y \\
* & X
\end{array}\right] \geq 0, \quad Z_{t t} \leq\left(u_{\lim }^{t}\right)^{2}} \\
& {\left[\begin{array}{cc}
X & * \\
C\left(r_{k}\right) \theta_{l}\left(r_{k}\right) & M
\end{array}\right] \geq 0, \quad M_{h h} \leq\left(y_{\text {lim }}^{h}\right)^{2},} \\
& {\left[\begin{array}{cc}
X & * \\
\theta_{l}\left(r_{k}\right) & X
\end{array}\right] \geq 0, \quad \forall r_{k+1} \in \pi_{r_{k}}^{u k}} \\
& {\left[\begin{array}{cccc}
\Pi_{r_{k}}^{k} X & U^{T}\left(r_{k}\right) & X Q^{1 / 2}\left(r_{k}\right) & Y^{T} Q^{1 / 2}\left(r_{k}\right) \\
* & W\left(r_{k+1}\right) & 0 & 0 \\
* & * & \gamma_{k} I & 0 \\
* & * & * & \gamma_{k} I
\end{array}\right] \geq 0,} \\
& \forall r_{k+1} \in \pi_{r_{k}}^{k}
\end{aligned}
$$

then the mode-independent state-feedback law can minimize the upper bound $\gamma_{k}$ on the objective function $J_{\infty}(k)$ and stabilize the closed-loop system in the ellipsoid $\varepsilon=\left\{x_{k}^{T} X^{-1} x_{k} \leq\right.$ $1\}$ and it is obtained by $u(k+i \mid k)=F x_{k+i \mid k}, F=Y X^{-1}$, where $X=\gamma_{k} P^{-1}, \theta_{l}\left(r_{k}\right)=A_{l}\left(r_{k}\right) X+B_{l}\left(r_{k}\right) Y, U^{T}\left(r_{k}\right)=$ $\left[\sqrt{\kappa_{r_{k}}^{1}} \theta_{l}^{T}\left(r_{k}\right), \ldots, \sqrt{\kappa_{r_{k}}^{\tau}} \theta_{l}^{T}\left(r_{k}\right)\right], W\left(r_{k+1}\right)=\operatorname{diag}\{X, X, \ldots, X\}$, $Z_{t t}$ and $M_{h h}$, respectively, describe the th and hth diagonal element of $Z, M$, and $u_{\mathrm{lim}}^{t}$ and $y_{\mathrm{lim}}^{h}$, respectively, describe the th and hth element of input and output constraints, $t=$ $1,2, \ldots, n_{u}, h=1,2, \ldots, n_{y}$.

3.2. Simplified MPC Design. In this section, a simplified MPC for uncertain MJS (1) is developed based on the online algorithm in Theorem 4; Figure 1 shows the simplified MPC schematic diagram. Then the simplified mode-independent feedback controller is designed regardless of model uncertainty and TP uncertainty since much more constraints will be nonactive in the neighboring region of origin and this freedom of feasibility is applied to improve the procedure of controller design.

Theorem 6. Consider uncertain MJS (1) associated with an initial state $x_{0}$ satisfying $x_{0}^{T} Q_{0}^{-1}\left(r_{0}\right) x_{0} \leq 1$; the simplified $M P C$ Algorithm 7 robustly stabilizes the closed-loop system.

Proof. For the $N$-step implementation at $x_{j}, j=1, \ldots, N$, the selection for $x_{j}$ in Algorithm 7 implies $Q_{j-1}^{-1}\left(r_{k}\right)<$ $Q_{j}^{-1}\left(r_{k}\right)$, which means the constructed ellipsoid $\xi_{j}=\{x \mid$ 


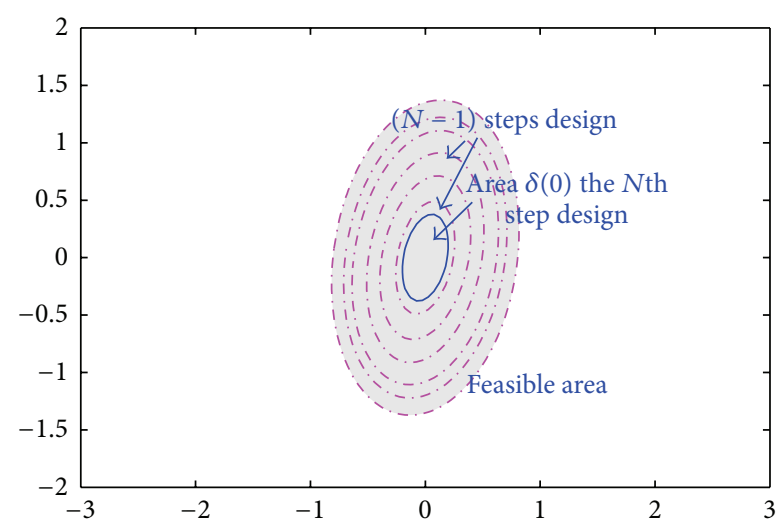

FIGURE 1: Simplified MPC schematic diagram.

$\left.x^{T} Q_{j}^{-1}\left(r_{k}\right) x \leq 1\right\}$ is embedded in $\xi_{j-1}$, that is, $\xi_{j} \subset \xi_{j-1}$. For a settled $x, \xi_{j}=\left\{x \mid x^{T} Q_{j}^{-1}\left(r_{k}\right) x \leq 1\right\}$ is decreasing monotonically associated with $j$, which guarantees the unique search in the search table for the largest $j$ for $\xi_{j}=\left\{x \mid x^{T} Q_{j}^{-1}\left(r_{k}\right) x \leq 1\right\}$. If $x_{k}$ belongs to $\xi_{j}=\left\{x \mid x^{T} Q_{j}^{-1}\left(r_{k}\right) x \leq 1\right\}$ and $\xi_{j+1}=$ $\left\{x \mid x^{T} Q_{j+1}^{-1}\left(r_{k}\right) x>1\right\}, j=1, \ldots, N-1$, by applying Theorem 3, the control law $u_{k}=F_{j}\left(r_{k}\right) x_{k}$ will steer the state in $\xi_{j-1}$ to $\xi_{j}$. Finally, the controller $u_{k}=F_{N} x_{k}$ (applying Corollary 5) make the state to be in $\xi_{N}$ and converge to the origin. Furthermore, the LP programming algorithm is utilized to remove redundant constraints [20] and construct a sequence of polyhedral invariant set for MJS and thus enlarge the feasible domain.

Algorithm 7 (simplified MPC applying polyhedral invariant set). Simplified MPC design is as follows.

(1) Select $x_{j}, j=1, \ldots, N$, which satisfy $\varepsilon_{j+1} \subset \varepsilon_{j}, \varepsilon_{N}=$ $\delta(0)$.

(2) For step 1 to $N-1$, calculate the corresponding modedependent gains $\gamma_{j}\left(r_{k}\right), Q_{j}\left(r_{k}\right), X_{j}\left(r_{k}\right), Y_{j}\left(r_{k}\right), F_{j}\left(r_{k}\right)$ by applying Theorem 4 and store them in a search table.

(3) For each $F_{j}\left(r_{k}\right)$, construct the corresponding polyhedral invariant set by the following algorithm: let $S_{j}\left(r_{k}\right)=\left[C^{T}\left(r_{k}\right),-C^{T}\left(r_{k}\right), F_{j}^{T}\left(r_{k}\right),-F_{j}^{T}\left(r_{k}\right)\right]^{T}, d_{j}\left(r_{k}\right)=$ $\left[y_{\max }^{T}\left(r_{k}\right), y_{\min }^{T}\left(r_{k}\right), u_{\max }^{T}\left(r_{k}\right), u_{\min }^{T}\left(r_{k}\right)\right]^{T}$. Select row $m$ from $\left(S_{j}\left(r_{k}\right), d_{j}\left(r_{k}\right)\right)$ and then check $\forall j$ if $S_{j, m}\left(r_{k}\left(A_{j}\left(r_{k}\right)+B_{j} F_{j}\right)\left(r_{k}\right) \leq d_{j, m}\left(r_{k}\right)\right)$ is redundant through solving the Linear programming:

$\max \rho_{j, m}$

s.t. $\rho_{j, m}=S_{j, m}\left(r_{k}\right)\left(A_{j}\left(r_{k}\right)+B_{j} F_{j}\left(r_{k}\right)\right) x-d_{j, m}\left(r_{k}\right)$

$S_{j}\left(r_{k}\right) x \leq d_{j}\left(r_{k}\right)$.

If $\rho_{j, m}>0$, it implies that the constraint $S_{j, m}\left(r_{k}\left(A_{j}\left(r_{k}\right)+B_{j} F_{j}\right)\left(r_{k}\right) \leq d_{j, m}\left(r_{k}\right)\right)$ is nonredundant; then renew the nonredundant
TABLE 1: The partly unknown TP matrix.

\begin{tabular}{lccc}
\hline 1 & 2 & 3 & 4 \\
\hline 0.361 & $?$ & 0.092 & $?$ \\
$?$ & 0.090 & $?$ & 0.248 \\
0.162 & 0.489 & $?$ & $?$ \\
$?$ & $?$ & 0.251 & $?$ \\
\hline
\end{tabular}

constraints as $S_{j}\left(r_{k}\right)=\left[S_{j}^{T}\left(r_{k}\right),\left(S_{j, m}\left(r_{k}\right)\left(A_{j}\left(r_{k}\right)+\right.\right.\right.$ $\left.\left.\left.B_{j}\left(r_{k}\right) F_{j}\left(r_{k}\right)\right)\right)^{T}\right]^{T}, d_{j}\left(r_{k}\right)=\left[d_{j}\left(r_{k}\right)^{T}, d_{j, m}\left(r_{k}\right)^{T}\right]^{T}$.

(4) Online implementation: search the state in the search table to fix the needed index $j(j<N)$, decide the smallest polyhedral invariant set $\chi_{j}\left(r_{k}\right)=\{x \mid$ $\left.S_{j}\left(r_{k}\right) x \leq d_{j}\left(r_{k}\right)\right\}$, and finally implement $u_{k}=$ $F_{j}\left(r_{k}\right) x_{k}$.

(5) Online implementation: continue to check if $x \in$ $\chi_{N}\left(r_{k}\right)=\left\{x \mid S_{N}\left(r_{k}\right) x \leq d_{N}\left(r_{k}\right)\right\}$ is satisfied; if it is true, then apply $u_{k}=F_{N} x_{k}$.

Remark 8. It should be noted that the more approximation of optimality can be obtained as $N$ increases; here $N$ can be chosen according to different prior requirements. Thus, we can adjust the numbers of design step in terms of different requirements.

\section{Illustrative Example}

Consider the discrete-time MJS with four modes $(\sigma=4)$ :

$$
\begin{array}{rlrl}
A_{11} & =\left[\begin{array}{cc}
1 & 0.1 \\
0.01 & 0.99
\end{array}\right], & B_{11}=\left[\begin{array}{c}
0.1 \\
0.187
\end{array}\right], \\
A_{12}=\left[\begin{array}{cc}
1 & 0.1 \\
0 & 0.05
\end{array}\right], & B_{12}=\left[\begin{array}{c}
0.1 \\
0.187
\end{array}\right], \\
A_{21}=\left[\begin{array}{cc}
1 & 0.1 \\
-0.1 & 0.99
\end{array}\right], & B_{21}=\left[\begin{array}{c}
0.1 \\
0.187
\end{array}\right], \\
A_{22}=\left[\begin{array}{cc}
1 & 0.1 \\
0.1 & 0.05
\end{array}\right], & B_{22}=\left[\begin{array}{c}
0.1 \\
0.187
\end{array}\right], \\
A_{31}=\left[\begin{array}{cc}
1 & 0.1 \\
0.2 & 0.99
\end{array}\right], & B_{31}=\left[\begin{array}{c}
0.1 \\
0.187
\end{array}\right], \\
A_{32}=\left[\begin{array}{cc}
1 & 0.1 \\
0.15 & 0.1
\end{array}\right], & B_{32}=\left[\begin{array}{c}
0.1 \\
0.187
\end{array}\right], \\
A_{41}=\left[\begin{array}{cc}
1 & 0.1 \\
0.05 & 0.5
\end{array}\right], & B_{41}=\left[\begin{array}{c}
0.1 \\
0.187
\end{array}\right], \\
A_{42}=\left[\begin{array}{cc}
1 & 0.1 \\
0.05 & 0.1
\end{array}\right], & B_{42}=\left[\begin{array}{c}
0.1 \\
0.187
\end{array}\right] .
\end{array}
$$

The detailed constraints are $u_{\max }=2$ and $y_{\max }=1.5$, initial state $x_{0}$ is $\left[\begin{array}{ll}-0.65 & 1\end{array}\right]^{T}$, and $C\left(r_{k}\right)=\left[\begin{array}{ll}1 & 0 \\ 0 & 1\end{array}\right]$. The positive definite weighting matrices are $Q\left(r_{k}\right)=\left[\begin{array}{ll}1 & 0 \\ 0 & 1\end{array}\right]$ and $R\left(r_{k}\right)=0.00002$. The partly unknown TP matrix is randomly generated in Table 1. 


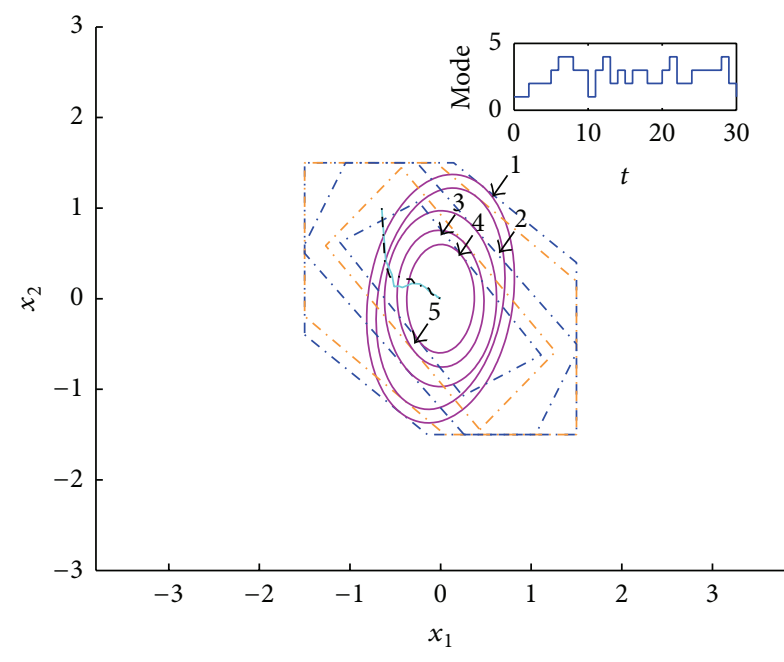

State of online algorithm

..- State of 5-step algorithm

FIGURE 2: Trajectory of system states.

TABLE 2: 20 times' average of 30 iterations of system state.

\begin{tabular}{lcc}
\hline Algorithm & 20 times' average & Variance \\
\hline Online & $11.6779 \mathrm{~s}$ & 0.0069 \\
5-step & $0.0055 \mathrm{~s}$ & $1.3173 e-006$ \\
\hline
\end{tabular}

Here we will show the 5-step example of the proposed Algorithm 7. Firstly, a state set $\left\{x_{j} \mid x_{j}=(0.5,-0.9),(0.4\right.$, $-0.8),(0.3,-0.7),(0.2,-0.6),(0.1,-0.5)\}$ is designed to compute the corresponding feedback gains $F_{j}\left(r_{k}\right)$. It is noted that the sequence of states $x_{j}$ guarantees that the constructed polyhedral invariant sets are embedded, that is, $S_{j} \subset S_{j-1}$. In this example, the first four mode-dependent feedback laws $F_{j}\left(r_{k}\right), j=1, \ldots, 4$ are obtained. When the state goes into the smallest polyhedral invariant set, the final-step (the fifthstep) gain $F_{5}$ is designed to steer the state to the origin regardless of model uncertainty and TP uncertainty.

For each chosen $x_{j}$ in Figure 2, the 5-step ellipsoid invariant sets (purple solid lines) and 5-step polyhedral invariant sets (blue and orange alternant dot dash lines) are illustrated using the numbers 1 to 5 . The stabilizable region of polyhedral invariant set constructed by Algorithm 7 is dramatically larger than that of ellipsoid invariant set while the dynamic response of simplified algorithm is comparable with online algorithm.

The results are computed at the same platform (AMD 2.1 GHz, memory 3.0 GB and MATLAB R2010a); the average time and variances of 30 times' running of the system are shown in Table 2. From the table, the burden of computation is significantly reduced by simplified algorithm.

\section{Conclusions}

The problem of simplified predictive controller design for MJS with mixed uncertainties is investigated. The simplified algorithm drastically reduces the online computational burden with only a little loss of performance. A numerical example is provided to illustrate the validity of the results.

\section{Conflict of Interests}

The authors declare that there is no conflict of interests regarding the publication of this paper.

\section{Acknowledgments}

This work was partially supported by the National Natural Science Foundation of China (61273087), the 111 Project (B12018), the Fundamental Research Funds for the Central Universities (JUSRP11459), the Program for Excellent Innovative Team of Jiangsu Higher Education Institutions, and the Fundamental Research Funds for the Central Universities (JUDCF12029).

\section{References}

[1] Z. Xiang, R. Wang, and Q. Chen, "Robust reliable stabilization of stochastic switched nonlinear systems under asynchronous switching," Applied Mathematics and Computation, vol. 217, no. 19, pp. 7725-7736, 2011.

[2] Z. Xiang, C. Qiao, and M. S. Mahmoud, "Finite-time analysis and $H_{\infty}$ control for switched stochastic systems," Journal of the Franklin Institute, vol. 349, no. 3, pp. 915-927, 2012.

[3] Z. Xiang, R. Wang, and Q. Chen, "Robust stabilization of uncertain stochastic switched nonlinear systems under asynchronous switching," Proceedings of the IMechE, Part I: Journal of Systems and Control Engineering, vol. 225, no. 1, pp. 8-20, 2011.

[4] E. K. Boukas, "Static output feedback control for stochastic hybrid systems: LMI approach," Automatica, vol. 42, no. 1, pp. 183-188, 2006.

[5] H. Gao, J. Lam, S. Xu, and C. Wang, "Stabilization and $H_{\infty}$ control of two-dimensional Markovian jump systems," IMA Journal of Mathematical Control and Information, vol. 21, no. 4, pp. 377-392, 2004.

[6] S. He and F. Liu, "Robust peak-to-peak filtering for Markov jump systems," Signal Processing, vol. 90, no. 2, pp. 513-522, 2010.

[7] S. He and F. Liu, "Finite-time $H_{\infty}$ control of nonlinear jump systems with time-delays via dynamic observer-based state feedback," IEEE Transactions on Fuzzy Systems, vol. 20, no. 4, pp. 605-614, 2012.

[8] S. He and F. Liu, "Finite-time boundedness of uncertain timedelayed neural network with Markovian jumping parameters," Neurocomputing, vol. 103, no. 1, pp. 87-92, 2013.

[9] Internet traffic report, 2008, http://www.internettracreport .com.

[10] L. Zhang and E.-K. Boukas, " $H_{\infty}$ control for discrete-time Markovian jump linear systems with partly unknown transition probabilities," International Journal of Robust and Nonlinear Control, vol. 19, no. 8, pp. 868-883, 2009.

[11] J. Xiong, J. Lam, H. Gao, and D. W. C. Ho, "On robust stabilization of Markovian jump systems with uncertain switching probabilities," Automatica, vol. 41, no. 5, pp. 897-903, 2005.

[12] Y. Yin, P. Shi, and F. Liu, "Gain-scheduled robust fault detection on time-delay stochastic nonlinear systems," IEEE Transactions on Industrial Electronics, vol. 58, no. 10, pp. 4908-4916, 2011. 
[13] J. B. R. do Val and T. Basar, "Receding horizon control of Markov jump linear systems," in Proceedings of the American Control Conference, pp. 3195-3199, Albuquerque, NM, USA, June 1997.

[14] B.-G. Park, J.-W. Lee, and W. H. Kwon, "Receding horizon control for linear discrete systems with jump parameters," in Proceedings of the 36th IEEE Conference on Decision and Control, pp. 3956-3957, San Diego, Calif, USA, December 1997.

[15] D. Q. Mayne, J. B. Rawlings, C. V. Rao, and P. O. M. Scokaert, "Constrained model predictive control: stability and optimality," Automatica, vol. 36, no. 6, pp. 789-814, 2000.

[16] L. Liu, Z. Liu, and J. Zhang, "Nonlinear model predictive control with terminal invariant manifolds for stabilization of underactuated surface vessel," Abstract and Applied Analysis, vol. 47, no. 4, pp. 861-864, 2011.

[17] J. A. De Doná, M. M. Seron, D. Q. Mayne, and G. C. Goodwin, "Enlarged terminal sets guaranteeing stability of receding horizon control," Systems \& Control Letters, vol. 47, no. 1, pp. 57-63, 2002.

[18] R. S. C. Lambert, P. Rivotti, and E. N. Pistikopoulos, "A novel approximation technique for online and multi-parametric model predictive control," Computer Aided Chemical Engineering, vol. 29, pp. 739-742, 2011.

[19] M. V. Kothare, V. Balakrishnan, and M. Morari, "Robust constrained model predictive control using linear matrix inequalities," Automatica, vol. 32, no. 10, pp. 1361-1379, 1996.

[20] B. Pluymers, J. A. Rossiter, J. A. K. Suykens, and B. De Moor, "The efficient computation of polyhedral invariant sets for linear systems with polytopic uncertainty," in Proceedings of the American Control Conference (ACC '05), pp. 804-809, June 2005. 


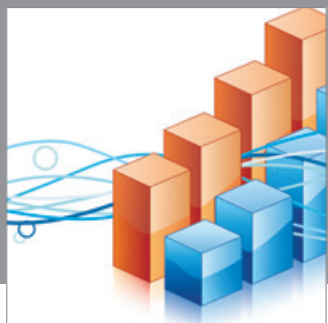

Advances in

Operations Research

mansans

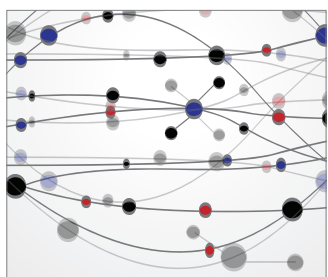

The Scientific World Journal
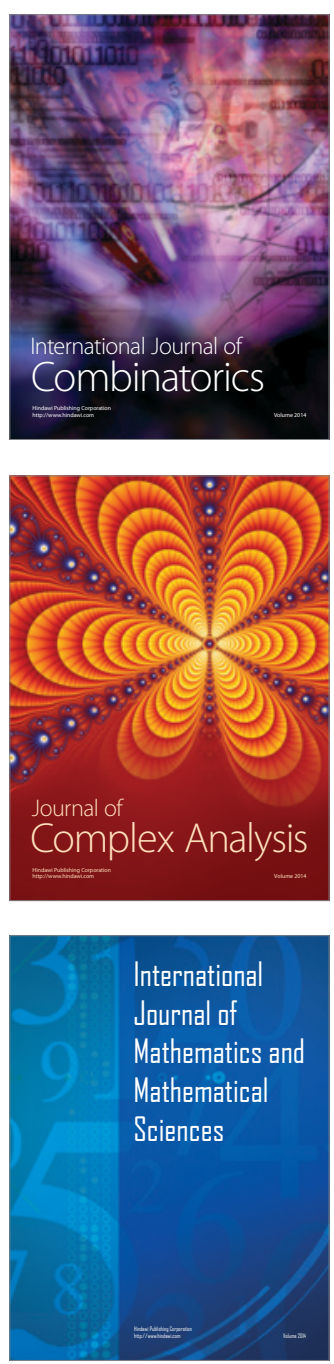
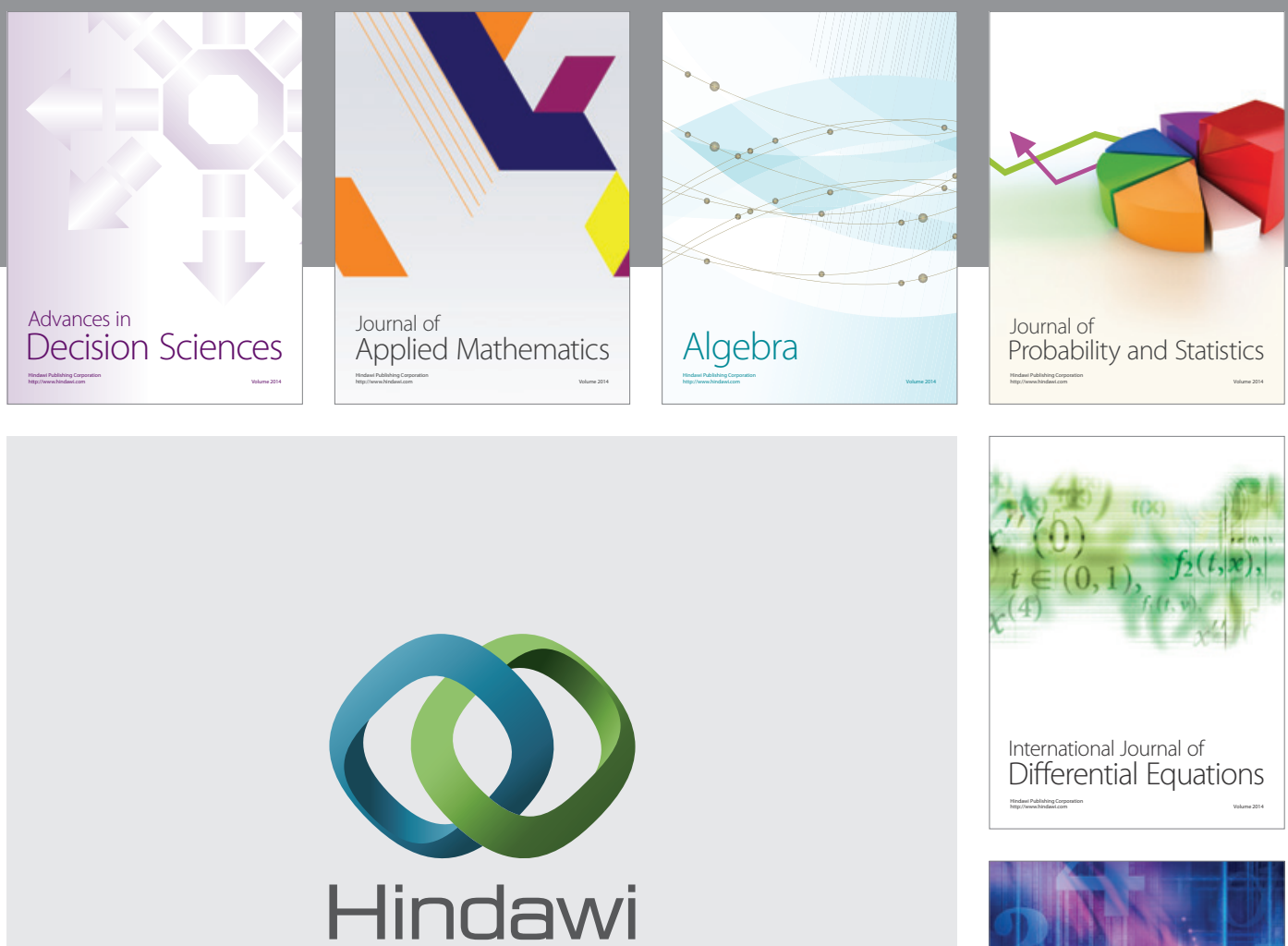

Submit your manuscripts at http://www.hindawi.com
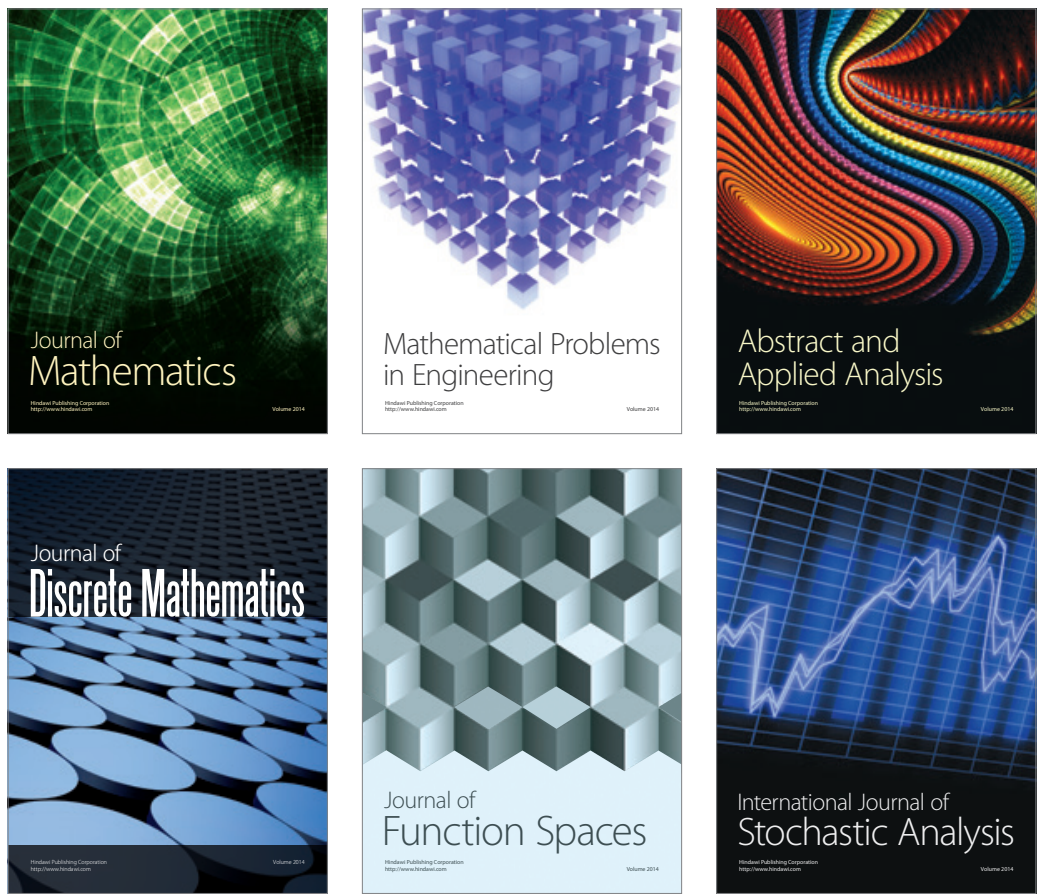

Journal of

Function Spaces

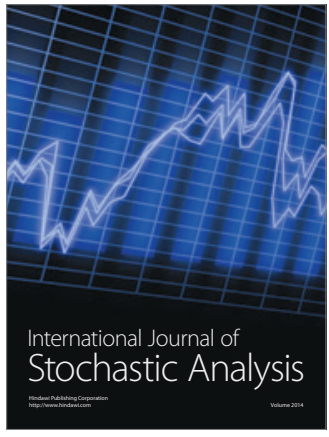

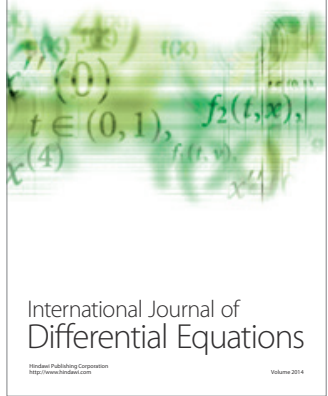
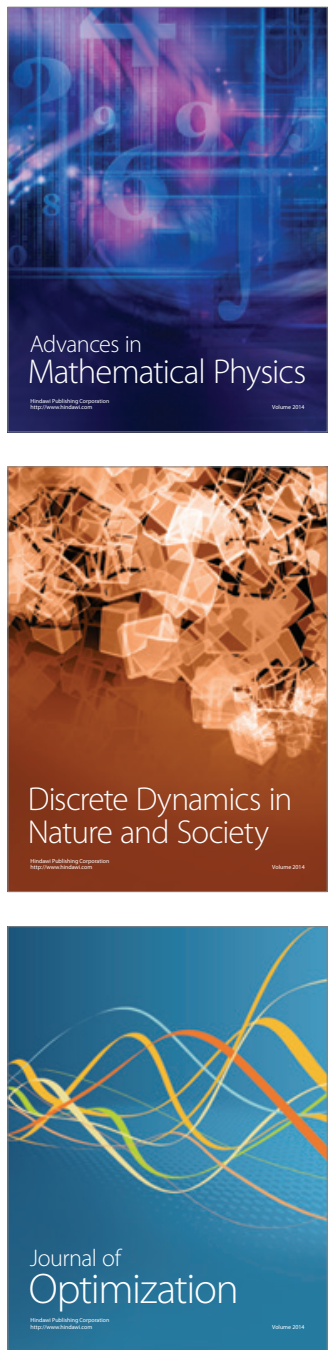